\title{
BMJ Open Clinical prediction models to diagnose neonatal sepsis: a scoping review protocol
}

Samuel R Neal (D) , ${ }^{1}$ David Musorowegomo, ${ }^{2}$ Hannah Gannon, ${ }^{3}$
Mario Cortina Borja, ${ }^{3}$ Michelle Heys,,${ }^{3,4}$ Gwen Chimhini, ${ }^{2}$ Felicity Fitzgerald ${ }^{1}$

To cite: Neal SR,

Musorowegomo D, Gannon H, et al. Clinical prediction models to diagnose neonatal sepsis: a scoping review protocol. BMJ Open 2020;10:e039712. doi:10.1136/ bmjopen-2020-039712

- Prepublication history and additional material for this paper are available online. To view these files, please visit the journal online (http://dx.doi. org/10.1136/bmjopen-2020039712).

SRN and DM contributed equally.

SRN and DM are joint first authors.

Received 23 April 2020 Revised 26 June 2020 Accepted 30 June 2020
Check for updates

(C) Author(s) (or their employer(s)) 2020. Re-use permitted under CC BY-NC. No commercial re-use. See rights and permissions. Published by BMJ.

For numbered affiliations see end of article.

Correspondence to Dr Felicity Fitzgerald; felicity.fitzgerald@ucl.ac.uk

\section{ABSTRACT}

Introduction Neonatal sepsis is responsible for significant morbidity and mortality worldwide. Diagnosis is often difficult due to non-specific clinical features and the unavailability of laboratory tests in many low-income and middle-income countries (LMICs). Clinical prediction models have the potential to improve diagnostic accuracy and rationalise antibiotic usage in neonatal units, which may result in reduced antimicrobial resistance and improved neonatal outcomes. In this paper, we outline our scoping review protocol to map the literature concerning clinical prediction models to diagnose neonatal sepsis. We aim to provide an overview of existing models and evidence underlying their use and compare prediction models between high-income countries and LMICs.

Methods and analysis The protocol was developed with reference to recommendations by the Joanna Briggs Institute. Searches will include six electronic databases (Ovid MEDLINE, Ovid Embase, Scopus, Web of Science, Global Index Medicus and the Cochrane Library) supplemented by hand searching of reference lists and citation analysis on included studies. No time period restrictions will be applied but only studies published in English or Spanish will be included. Screening and data extraction will be performed independently by two reviewers, with a third reviewer used to resolve conflicts. The results will be reported by narrative synthesis in line with the Preferred Reporting Items for Systematic reviews and Meta-Analyses extension for Scoping Reviews guidelines.

Ethics and dissemination The nature of the scoping review methodology means that this study does not require ethical approval. Results will be disseminated through peer-reviewed publications and conference presentations, as well as through engagement with peers and relevant stakeholders.

\section{INTRODUCTION}

Despite significant progress in global child health over the past two decades, there were 2.5 million neonatal deaths in 2018 with a global neonatal mortality rate of 18 deaths per 1000 live births. ${ }^{1}$ The vast majority of these deaths occur in low-income and middle-income countries (LMICs) and are most commonly due to prematurity (35\%), intrapartum-related complications (24\%)

\section{Strengths and limitations of this study}

- There have been few recent attempts to scope literature concerning clinical prediction models to diagnose neonatal sepsis across high-income and low-income and middle-income countries.

- The protocol was developed with reference to recommendations by the Joanna Briggs Institute and the review will be reported in line with the Preferred Reporting Items for Systematic reviews and MetaAnalyses extension for Scoping Reviews guidelines.

- The search strategy includes six electronic databases, hand searching of reference lists and citation analysis on included studies.

- A limitation of the review is that we will only include non-grey literature published in English or Spanish and it is possible that studies relevant to non-English or non-Spanish speaking settings will not be included.

and neonatal sepsis $(15 \%) .{ }^{1}$ Neonatal sepsis has an estimated global incidence of 2202 per 100000 live births and a global case fatality rate of between $11 \%$ and $19 \% .^{2}$ Moreover, it is a significant source of morbidity for survivors: complications including neurodevelopmental disorders, cerebral palsy and visual or hearing impairment may persist beyond the neonatal period. ${ }^{3}$ Therefore, addressing neonatal sepsis as a preventable and treatable cause of neonatal morbidity and mortality is a global priority.

Neonatal sepsis is a clinical syndrome that results from systemic infection in the first month of life. ${ }^{4}$ It is typically classified as early onset sepsis (EOS, onset within the first 48-72 hours of life) or late-onset sepsis (LOS, onset after the first 48-72 hours of life) to reflect the differing microbiology of these two disease patterns. ${ }^{5}$ EOS results from vertically transmitted infections with pathogens obtained from the maternal genital tract shortly before or during birth. ${ }^{6}$ Group B streptococcus (GBS) and Escherichia coli account for the majority of cases of EOS in 
high-income settings, and risk factors for these infections include prematurity, low birth weight, prolonged rupture of membranes, maternal fever during labour and maternal rectovaginal colonisation with GBS. ${ }^{6}$ In comparison, LOS occurs due to pathogens acquired through interaction with the home or hospital environment. ${ }^{7}$ Coagulase-negative staphylococci are the most common organisms of LOS and other major pathogens include Staphylococcus aureus, E. coli, Klebsiella species, Enterobacter species, Pseudomonas species and fungal infection with Candida species. ${ }^{7}$ It should be noted, however, that the exact microbiology of neonatal sepsis differs greatly across geographical regions and is liable to change over time. ${ }^{8}$ Furthermore, the microbiology is difficult to determine in settings with limited or no access to reliable culture methods. There is increasing recognition that the classification of neonatal sepsis as EOS or LOS is misplaced in LMICs. In these settings, babies are exposed from birth to organisms typically associated with LOS due to poor infection prevention and control practices such as hand hygiene, aseptic delivery and limited availability of GBS screening services in pregnancy. ${ }^{9}$ In a systematic review of data from sub-Saharan Africa, S. aureus (25\%) and Klebsiella species (21\%) were the most common causative organisms of neonatal sepsis. ${ }^{10}$ Thus, in LMICs, it may be sensible to label all infections in facility-born neonates as hospital acquired, even if the infection presents within the first few days of life. ${ }^{9}$

Diagnosis of neonatal sepsis is hindered by non-specific clinical features such as temperature instability, lethargy, poor feeding and respiratory distress, which often overlap with non-infectious diseases. The current gold standard method for diagnosing neonatal sepsis is the identification of a pathogenic organism from a normally sterile site (eg, blood or cerebrospinal fluid). ${ }^{5}$ However, clinical sepsis (where the infant shows clinical features of sepsis despite negative blood cultures) is a recognised entity and may be more common than blood culture-proven sepsis, especially in the context of previous antibiotic exposure in the baby or mother. ${ }^{11}$ When deciding to treat suspected cases of neonatal sepsis, there is a fine balance between failing to treat a serious infection and unnecessary use of antibiotics. Antimicrobial resistance is a growing global concern, with one estimate suggesting that $31 \%$ of annual sepsis-related neonatal deaths globally could be attributable to antimicrobial resistance. ${ }^{12}$ Furthermore, some reports have suggested that prolonged exposure to antibiotics is associated with negative neonatal outcomes such as death and necrotising enterocolitis. ${ }^{13}$ Therefore, accurately identifying infants with neonatal sepsis is vital to guide the optimal use of antibiotics, reduce antimicrobial resistance and improve neonatal outcomes.

\section{Study rationale}

Clinical prediction models are tools that combine multiple characteristics (or predictors) to estimate the probability of a diagnosis or prognostic outcome and they have gained increasing research attention in recent years. ${ }^{14}$ Multiple prediction models exist to estimate the risk of neonatal sepsis based on a wide range of clinical features, risk factors and/or laboratory tests, for example, the Kaiser Permanente neonatal sepsis risk calculator for EOS. ${ }^{15}$ Clinical prediction models for neonatal sepsis have the potential to improve diagnostic accuracy and rationalise antibiotic usage in neonatal units. Models that do not include laboratory results as predictors are of particular importance in LMICs where basic laboratory tests are often unavailable and the initial care and clinical management of newborn infants may fall to lower cadre healthcare workers in remote settings with limited senior support. Some models used in LMICs have high sensitivity but low specificity for neonatal sepsis and result in the overuse of antibiotics. ${ }^{16}$

A search to identify existing scoping reviews or systematic reviews of clinical prediction models to diagnose neonatal sepsis yielded three potentially relevant studies. Two reviews examined clinical prediction models for severe infections in children, however, both excluded those targeting neonates. ${ }^{17} 18$ One review examined clinical prediction models for healthcare-associated bloodstream infections in neonates, but excluded models developed for EOS and the searches are now relatively outdated in this rapidly evolving field. ${ }^{19}$ Therefore, the present review will provide an important summary of existing clinical prediction models to diagnose neonatal sepsis to form a basis for future primary research or systematic reviews.

\section{Study objectives}

The aim of this scoping review is to map the literature concerning clinical prediction models to diagnose neonatal sepsis in high-income countries (HICs) and LMICs.

The specific objectives are:

- To provide an overview of existing clinical prediction models to diagnose neonatal sepsis.

- To determine the evidence underlying the use of clinical prediction models to diagnose neonatal sepsis.

- To compare clinical prediction models to diagnose neonatal sepsis between HICs and LMICs.

- To identify unanswered research questions surrounding clinical prediction models to diagnose neonatal sepsis, which may guide future primary research or systematic reviews.

\section{METHODS AND ANALYSIS}

The methods for this review were developed with reference to the scoping review guidelines provided by the Joanna Briggs Institute. ${ }^{20}$ The major components of the review process are detailed ahead.

\section{Research question}

Our review will be guided by the following specific research questions (table 1 ). 
Table 1 Research questions

What clinical prediction models exist to diagnose neonatal sepsis?

What modelling methods are used to derive these models?
What predictors are used in
these models?

What questions remain unanswered regarding clinical prediction models to diagnose neonatal sepsis?

HIC, high-income countries; LMIC, low-income and middleincome country.

\section{Inclusion criteria}

The inclusion criteria (table 2) were formulated according to the 'Population-Concept-Context' framework recommended by the Joanna Briggs Institute. ${ }^{20}$ As a scoping review is an iterative process, these may be amended as the review progresses and the extent of the literature becomes apparent.

\section{Search strategy}

The following databases will be searched: Ovid MEDLINE and Epub Ahead of Print, In-Process and Other NonIndexed Citations and Daily; Ovid Embase; Scopus; Web of Science; Global Index Medicus and the Cochrane Library. Search terms were constructed to capture variations of 'neonate', 'sepsis' and 'prediction model' by examining the common text words and index terms of the known relevant literature. These keywords were further expanded by including relevant potential synonyms. The search strategy for Ovid MEDLINE is detailed in table 3 and a complete list of search strategies for each database can be found in the online supplementary appendix. It is anticipated that additional keywords and index terms may be identified as the review progresses, and these will be incorporated into the search strategy and the searches will be rerun as applicable. To identify additional studies not found through the primary database searches, citation analysis will be performed on included studies using the citation analysis function of Scopus, Web of Science and Google Scholar. Furthermore, the reference list of included studies will be hand searched. No time period or language restrictions will be applied to the search strategy, but studies will be manually limited to the English or Spanish language at the study selection stage to reflect

Table 2 Inclusion criteria

Population

\begin{tabular}{|c|c|}
\hline Concept & $\begin{array}{l}\text { Studies that develop, validate or assess the impact of a clinical prediction model to diagnose } \\
\text { neonatal sepsis. } \\
\text { Studies that report any of: } \\
\text { modelling methods (including participants, predictors, outcomes and type of model). } \\
\text { model performance (including sensitivity and specificity). } \\
\text { success of implementation (including acceptability and any changes to practice or } \\
\text { outcomes). } \\
\text { Only internally and/or externally validated models will be included. } \\
\text { Management algorithms, decision rules or prediction models based on expert opinion will not be } \\
\text { included unless validated in a subsequent study. } \\
\text { Studies evaluating prognostic models (eg, to predict neonatal sepsis-related mortality or morbidity) } \\
\text { will be excluded. }\end{array}$ \\
\hline Context & $\begin{array}{l}\text { Studies from any country. } \\
\text { Studies from any healthcare setting (including neonatal unit, emergency department, outpatient or } \\
\text { community setting). }\end{array}$ \\
\hline Types of studies & $\begin{array}{l}\text { Randomised and quasi-randomised controlled trials, cohort studies, cross-sectional studies, case- } \\
\text { control studies and guidelines. } \\
\text { Letters, comments and conference proceedings will be included if sufficient details are provided. } \\
\text { Studies published in English or Spanish. } \\
\text { Systematic reviews, meta-analyses and editorials will be excluded, but will be used to identify } \\
\text { relevant primary literature. }\end{array}$ \\
\hline
\end{tabular}

- Human neonates (aged <28 days of life or hospitalised to a neonatal unit) being evaluated for neonatal sepsis (as defined by the individual studies).

Studies examining a range of patient ages will be included, providing sufficient data are available to examine findings for neonates in isolation.

Studies that develop, validate or assess the impact of a clinical prediction model to diagnose al sepsis.

Studies that report any of:

model performance (including sensitivity and specificity) outcomes)

Only internally and/or externally validated models will be included.

included unless validated in a subsequent study.

Studies evaluating prognostic models (eg, to predict neonatal sepsis-related mortality or morbidity) 


\begin{tabular}{|c|c|}
\hline 1 & exp Infant, Newborn/ \\
\hline 2 & $\begin{array}{l}\text { (neonat* or newborn* or new born* or } \\
\text { baby or babies or premature or preterm } \\
\text { or infant* or low birth weight or LBW or } \\
\text { VLBW or ELBW or NICU*).ti,ab,kw. }\end{array}$ \\
\hline 3 & 1 or 2 \\
\hline 4 & exp Sepsis/ \\
\hline 5 & (sepsis or septic ${ }^{\star}$ or bacter?emia).ti,ab,kw \\
\hline 6 & 4 or 5 \\
\hline 7 & $\begin{array}{l}\text { Decision Support Techniques/ or Neonata } \\
\text { Screening/ }\end{array}$ \\
\hline 8 & 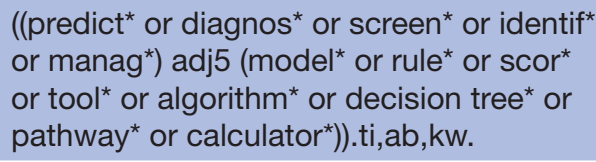 \\
\hline 9 & 7 or 8 \\
\hline 10 & 3 and 6 and 9 \\
\hline
\end{tabular}

ELBW, extremely low birth weight; LBW, low birth weight; NICU, neonatal intensive care unit; VLBW, very low birth weight.

the languages spoken by the review team. Searches will be updated prior to publication of our findings to ensure no recent studies are missed.

\section{Evidence selection}

All identified records will be imported into EndNote X9 (Clarivate Analytics, 2018) for bibliographic management and deduplication. First, titles and abstracts will be examined against the inclusion criteria (table 2) to determine whether the study is potentially eligible for inclusion. Next, full texts of potentially eligible studies will be obtained and examined to confirm their eligibility. Relevant authors will be contacted to request full texts if required. Record screening will be performed independently by two reviewers using the Rayyan web and mobile application. ${ }^{21}$ Studies in Spanish will be translated to English by $\mathrm{MCB}$ and then considered for inclusion. Conflicts will be resolved by a third reviewer and discussion among the review team. The first database searches were performed in December 2019 (identifying 2776 records after deduplication) and title and abstract screening is currently ongoing.

\section{Data extraction}

Data extraction will be performed independently by two reviewers. A draft data extraction form has been designed for this review and is shown in the online supplementary appendix. This form was adapted from a template provided by the Joanna Briggs Institute ${ }^{20}$ and will be further refined as the review progresses. Data to be extracted are based on the Transparent Reporting of a multivariable prediction model for Individual Prognosis or Diagnosis statement ${ }^{22}$ and include study author(s), year of publication and name of the prediction model described; sources of data, number and characteristics of study participants; type of model, predictors used and defined outcome; and model performance or other evidence regarding the use of the model in clinical practice.

\section{Analysis of the evidence and presentation of the results}

The results of this review will be reported by narrative synthesis in line with the recommendations set out in the Preferred Reporting Items for Systematic reviews and Meta-Analyses extension for Scoping Reviews. ${ }^{23}$ The characteristics of several subgroups of clinical prediction models will be compared, including:

- Those that were developed and validated for use in HICs versus LMICs, as defined by the World Bank classification. ${ }^{24}$

- Those for diagnosing specific subgroups of neonatal sepsis (eg, EOS vs LOS).

- Those based solely on clinical predictors versus those that require laboratory tests.

- Those that specifically consider the management of neonates born to mothers with chorioamnionitis.

- Those using different outcome definitions for neonatal sepsis (such as those defining sepsis as a positive blood culture vs those that also include 'clinical sepsis').

Data for quantitative outcomes such as model performance or measures of changes to clinical outcomes will not be pooled in a meta-analysis but, rather, general trends will be discussed. Furthermore, explicit risk of bias assessment will not be performed, as our aim is to report the extent of the current literature. It is hoped that this review can then act as a basis to determine important research questions for future primary research and systematic reviews or meta-analyses.

\section{Patient and public involvement}

This research was done without patient involvement. Patients were not invited to comment on the study design and were not consulted to develop patient-relevant outcomes or interpret the results. Patients were not invited to contribute to the writing or editing of this document for readability or accuracy.

\section{Ethics and dissemination}

As the scoping review methodology involves reviewing and collecting data from publicly available sources, this study does not require ethical approval. The results of this review will be disseminated through a peer-reviewed publication and/or conference presentation. Furthermore, we will engage with the stakeholders of our local and international projects to widen the dissemination of our findings. By identifying gaps in the literature, we hope that this review can form a basis for future primary research and systematic reviews or meta-analyses.

Author affiliations

${ }^{1}$ Infection, Immunity and Inflammation, UCL Great Ormond Street Institute of Child Health, University College London, London, UK 
${ }^{2}$ Department of Paediatrics and Child Health, University of Zimbabwe College of Health Sciences, Harare, Zimbabwe

${ }^{3}$ Population, Policy and Practice, UCL Great Ormond Street Institute of Child Health, University College London, London, UK

${ }^{4}$ Specialist Children's and Young People's Services, East London NHS Foundation Trust, London, UK

Acknowledgements The authors would like to thank Heather Chesters (Deputy Librarian at the UCL Great Ormond Street Institute of Child Health Library) for her advice and guidance when developing the search strategy for this review.

Contributors FF and MH conceived the idea for this review. SRN developed the research question, study methods and drafted the manuscript. FF, MH, GC and MCB supervised the protocol design and continue to supervise the review process. SRN, $\mathrm{DM}$ and $\mathrm{HG}$ piloted the protocol and continue to screen records for inclusion. All authors discussed the protocol design and critically edited and approved the final manuscript. GC and FF contributed equally as last authors.

Funding This research received no specific grant from any funding agency in the public, commercial or not-for-profit sectors but was supported by the National Institute for Health Research (NIHR) Great Ormond Street Hospital Biomedical Research Centre. FF is supported by the Academy of Medical Sciences, the funders of the Starter Grant for Clinical Lecturers scheme and the NIHR Great Ormond Street Hospital Biomedical Research Centre. The views expressed are those of the authors and not necessarily those of the National Health Service (NHS), the NIHR or the UK Department of Health.

Competing interests None declared.

Patient and public involvement Patients and/or the public were not involved in the design, or conduct, or reporting, or dissemination plans of this research.

Patient consent for publication Not required.

Provenance and peer review Not commissioned; externally peer reviewed.

Open access This is an open access article distributed in accordance with the Creative Commons Attribution Non Commercial (CC BY-NC 4.0) license, which permits others to distribute, remix, adapt, build upon this work non-commercially, and license their derivative works on different terms, provided the original work is properly cited, appropriate credit is given, any changes made indicated, and the use is non-commercial. See: http://creativecommons.org/licenses/by-nc/4.0/.

ORCID iD

Samuel R Neal http://orcid.org/0000-0001-6832-9839

\section{REFERENCES}

1 United Nations Inter-agency Group for Child Mortality Estimation. Levels \& trends in child mortality: report 2019. New York: United Nations Children's Fund, 2019.

2 Fleischmann-Struzek C, Goldfarb DM, Schlattmann P, et al. The global burden of paediatric and neonatal sepsis: a systematic review. Lancet Respir Med 2018;6:223-30.

3 Seale AC, Blencowe H, Zaidi A, et al. Neonatal severe bacterial infection impairment estimates in South Asia, sub-Saharan Africa, and Latin America for 2010. Pediatr Res 2013;74 Suppl 1:73-85.

4 Nizet V, Klein JO. Bacterial sepsis and meningitis. In: Wilson CB, Nizet V, Maldonado YA, eds. Remington and Klein's infectious diseases of the fetus and newborn infant. 8th edn. Philadelphia: Elsevier Saunders, 2016: 217-71.

5 Shane AL, Sánchez PJ, Stoll BJ. Neonatal sepsis. Lancet 2017;390:1770-80.

6 Simonsen KA, Anderson-Berry AL, Delair SF, et al. Early-onset neonatal sepsis. Clin Microbiol Rev 2014;27:21-47.

7 Dong Y, Speer CP. Late-onset neonatal sepsis: recent developments. Arch Dis Child Fetal Neonatal Ed 2015;100:F257-63.

8 Zaidi AK, Darmstadt GL, Stoll BJ. Neonatal infections: a global perspective. In: Wilson CB, Nizet V, Maldonado YA, eds. Remington and Klein's infectious diseases of the fetus and newborn infant. 8th edn. Philadelphia: Elsevier Saunders, 2016: 24-53.

9 Zaidi AKM, Huskins WC, Thaver D, et al. Hospital-acquired neonatal infections in developing countries. Lancet 2005;365:1175-88.

10 Okomo U, Akpalu ENK, Le Doare K, et al. Aetiology of invasive bacterial infection and antimicrobial resistance in neonates in sub-Saharan Africa: a systematic review and meta-analysis in line with the STROBE-NI reporting guidelines. Lancet Infect Dis 2019;19:1219-34.

11 Wynn JL, Wong HR, Shanley TP, et al. Time for a neonatalspecific consensus definition for sepsis. Pediatr Crit Care Med 2014;15:523-8

12 Laxminarayan R, Matsoso P, Pant S, et al. Access to effective antimicrobials: a worldwide challenge. Lancet 2016;387:168-75.

13 Dona D, Mozzo E, Mardegan V, et al. Antibiotics prescriptions in the neonatal intensive care unit: how to overcome everyday challenges. Am J Perinatol 2017;34:1169-77.

14 Steyerberg EW. Clinical prediction models. 1st edn. New York: Springer, 2009

15 Kuzniewicz MW, Walsh EM, Li S, et al. Development and implementation of an early-onset sepsis calculator to guide antibiotic management in late preterm and term neonates. Jt Comm J Qual Patient Saf 2016;42:232-9.

16 Chimhini G, Chimhuya S, Madzudzo L, et al. Auditing use of antibiotics in Zimbabwean neonates. Infection Prevention in Practice 2020;2:100046.

17 Keitel K, Kilowoko M, Kyungu E, et al. Performance of prediction rules and guidelines in detecting serious bacterial infections among Tanzanian febrile children. BMC Infect Dis 2019;19:769.

18 Thompson M, Van den Bruel A, Verbakel J, et al. Systematic review and validation of prediction rules for identifying children with serious infections in emergency departments and urgent-access primary care. Health Technol Assess 2012;16:1-100.

19 Verstraete EH, Blot K, Mahieu L, et al. Prediction models for neonatal health care-associated sepsis: a meta-analysis. Pediatrics 2015;135:e1002-14.

20 Joanna Briggs Institute. Development of a scoping review protocol 2019. Available: https://wiki.joannabriggs.org/display/MANUAL/11. 2+Development+of+a+scoping+review+protocol. [Accessed $4 \mathrm{Apr}$ 2020].

21 Ouzzani M, Hammady H, Fedorowicz Z, et al. Rayyan-a web and mobile app for systematic reviews. Syst Rev 2016;5:210.

22 Moons KGM, Altman DG, Reitsma JB, et al. Transparent reporting of a multivariable prediction model for individual prognosis or diagnosis (TRIPOD): explanation and elaboration. Ann Intern Med 2015;162:W1-73.

23 Tricco AC, Lillie E, Zarin W, et al. PRISMA extension for scoping reviews (PRISMA-ScR): checklist and explanation. Ann Intern Med 2018;169:467-73.

24 The World Bank. World bank country and lending groups 2019. Available: https://datahelpdesk.worldbank.org/knowledgebase/ articles/906519 [Accessed 4 Apr 2020]. 